\title{
Chanter, enchanter en Grèce ancienne.
}

À propos de Sappho, femme poète et dixième Muse

\section{Ana Iriarte}

\section{(2) OpenEdition \\ Journals}

Édition électronique

URL : https://journals.openedition.org/clio/2182

DOI : $10.4000 /$ clio. 2182

ISSN : 1777-5299

Éditeur

Belin

Édition imprimée

Date de publication : 1 avril 2007

ISBN : 978-2-85816-900-9

ISSN : $1252-7017$

Référence électronique

Ana Iriarte, "Chanter, enchanter en Grèce ancienne. », Clio. Histoire, femmes et sociétés [En ligne], 25 |

2007, mis en ligne le 19 décembre 2008, consulté le 22 avril 2022. URL : http://

journals.openedition.org/clio/2182 ; DOI : https://doi.org/10.4000/clio.2182

Ce document a été généré automatiquement le 22 avril 2022

Tous droits réservés 


\title{
Chanter, enchanter en Grèce ancienne.
}

\author{
À propos de Sappho, femme poète et dixième Muse
}

\section{Ana Iriarte}

\author{
Voici venir l'heure du prestige pour la race des \\ femmes (timà gunaikeío génei) ; \\ une injurieuse renommée (duskélados pháma) ne \\ pèsera plus sur elles. \\ Les poèmes des antiques chanteurs cesseront de \\ célébrer ma perfidie. Phoibos, le maître des \\ mélodies, n'a point doté notre esprit du chant \\ inspiré de la lyre (lúras ... théspin aoidàn) ; \\ sans quoi j'aurais retourné l'hymne contre la race \\ des mâles ${ }^{1}$.
}

1 En 431 av. J.C., ces vers du poète Euripide sont chantés à l'unisson sur la scène du théâtre de Dionysos par les quinze Grecques qui forment le chœur de Médée. Nous sommes au printemps de l'année qui marquera et le déclenchement irréversible de la guerre du Péloponnèse et le début du déclin de la demokratia athénienne ; régime dont le verbe musical du polémique et polémiste Euripide ne cesse de signaler les contradictions.

2 C'est sans doute en pensant à une tradition poétique antérieure, très solidement établie, concernant "l'injurieuse renommée» des femmes qu'Euripide compose la plainte revendicatrice que nous venons de citer. Cette tradition - comme l'a amplement constaté la critique ${ }^{2}$ - représentée à merveille par le célèbre "iambe des femmes » de Sémonide d'Amorgos, inclut également bien d'autres "antiques chanteurs» qui, comme l'Odyssée ${ }^{3}$ ou l'inventeur de Pandore ${ }^{4}$, célèbrent la perfidie de la Femme, notamment en la présentant comme un piège pour les hommes; en réalité le plus terrible des pièges, parce que le seul vraiment inévitable pour leur survie.

3 Nous n'avons pas l'intention d'insister dans cet article sur l'aspect relatif à la «mauvaise renommée » que la « race des femmes » doit aux anciens poètes. Toutefois, 
cette partie de la plainte du chœur énonce un conflit entre l'univers féminin et la musique ancestrale, conflit fort révélateur à l'heure de prendre contact avec le monde des musiciennes en pays grec. En effet, qu'en est-il de l'assertion "Phoibos n'a point doté notre esprit du chant inspiré de la lyre »?

4 Certains commentateurs de ce vers constatent qu'Euripide "chooses to ignore" l'existence de célébrités comme Sappho de Lesbos ${ }^{5}$. Et ce « choix » a été aussi interprété comme une dévalorisation des poétesses, telle Corinne de Tanagra, Praxilla de Sicyone, Télésilla d'Argos et Sappho elle-même, face aux poètes mâles de l'archaïsme ${ }^{6}$. Or, à notre avis, l'assertion du poète tragique prend tout son sens si, en la situant dans le moment historique où elle a vu le jour ${ }^{7}$, on l'entend comme l'évocation d'un fait incontestable, à savoir que le siècle d'or de la démocratie athénienne, sommet de la civilisation grecque, n’a pas légué à la postérité un seul nom de musicienne célèbre.

\section{Musique et citoyenneté}

5 Conçue comme un ensemble incluant la technique instrumentale et la danse, la tradition littéraire et philosophique, la mousiké, la musique,synonyme de culture, constituait le point fort de la paideia,l'éducation en pays grec. "La paideía est tout d'abord l'œuvre des Muses et d'Apollon ", dit littéralement Platon", juste avant de soutenir que celui qui est achóreutos (étranger aux chants et danses des chœurs) ne peut qu'être considéré comme apaídeutos (in-éduqué, ignorant).

6 Dans l'Athènes démocratique, l'étude du chant et de la lyre faisait partie de la formation conduisant les fils des citoyens à incarner la célèbre kalokagathía, l'idéal grec de virilité qui unit beauté et bonté9. À l'âge adulte, ces privilégiés exhibaient leur art lors des très fréquentes célébrations des banquets privés ou publics, et lorsqu'ils prenaient part dans les chœurs des tragédies, étant donné que ces chœurs - témoins, dit Reinach «de la floraison des études musicales dans la démocratie attique depuis l'époque des Pisistratides jusqu'à la guerre du Péloponnèse $»^{10}$ - étaient composés par des citoyens, même lorsqu'il s'agissait de rôles féminins.

7 Comme de nos jours l'opéra chinois, le théâtre attique était uniquement représentépar des acteurs, choreutes et musiciens, tous hommes, et, selontoute probabilité, pour un public exclusivement masculin. En effet, ce genre, qui est, par excellence, celui de la démocratie, n'autorisait pas la présence des femmes sur scène. Or - paradoxalement ? il situe le féminin au cœur du spectacle: sur la scène tragique, la voix aiguë des femmes ${ }^{11}$ - de nombreuses "voix endeuillées ", celle de Cassandre devant les anciens d'Argos, celle d'Antigone face à Créon, ou celle des compagnes de Médée face aux "antiques chanteurs" - s'érige en protagoniste pour faire face au grave discours politique, lui rappelant la dimension conflictuelle qu'il comporte ${ }^{12}$. Sans aucun doute la voix féminine est l'un des fondements du drame classique - drame éminemment musical dont l'opéra moderne ne serait que « la caricature » d'après le jugement sévère de Nietzsche. Mais cette voix toujours mimée par des acteurs mâles, ne donna pas naissance à la figure historique de la diva, de la cantatrice.

8 L'institution des banquets - qui brilla autant que le théâtre dans l'Athènes de l'époque classique - fut, pour sa part, plus accueillante vis-à-vis du versant artistique de "la race des femmes" car elle intégrait un certain type de musiciennes. Dans ces actes sociaux, la musique exécutée par des femmes pour le plaisir des invités (hommes libres uniquement) occupait une place importante, témoin la joueuse de flûte, figure centrale 
dans les représentations aussi bien littéraires qu'iconographiques desbanquets ${ }^{13} \cdot \mathrm{La}$ flûte - instrumentqui éveille l'enthousiasme dionysiaque ${ }^{14}$, qui peut " enchanter » sans accompagner le chant - était à tel point associée aux femmes requises dans les banquets, qu'elle devint le symbole même de la prostituée.

Quant aux mères, filles et épouses légitimes des citoyens athéniens de l'époque classique, elles vivaient à l'écart de ces rencontres où régnaient vin et musique. Ce qui ne veut pas dire que ces honnêtes femmes aient étéexclues de l'univers musical dans son ensemble. L'iconographie de l'âge d'or athénien - observatoire incomparable pour l'étude de l'espace domestique que les sources littéraires tendent à entourer de silence - révèle sans ambiguïté la présence d'une activité musicale au sein du gynécée. Activité celle-ci liée exclusivement, il est vrai, à la lyre ${ }^{15}$, la grave lyre apollinienne, support de la mémoire collective précédant l'avènement de la historía proprement dite, et support, en plein classicisme, de la sage parole poétique qui aide à reconstituer la bonne mémoire au lieu de provoquer l'exaltation, indépendante de la parole, caractéristique de la flûte ${ }^{16}$. Autrement dit, les foyers des citoyens raffinés d'Athènes abritaient des femmes parfaitement cultivées, bien que la polis démocratique n'offrît jamais à ces dames la possibilité de montrer leurs connaissances en public ${ }^{17}$, et encore moins, d'immortaliser leur nom en participant aux concours poétiques ${ }^{18}$.

Anonymat des dames auxquelles on interdit l'exhibition de leurstalents artistiques en public, étrangeté dionysiaque des voix aiguës des héroïnes tragiques, son également aigu des flûtes que des virtuoses inconnues jouent dans les banquets, tel est le sort que la démocratie athénienne réserve aux musiciennes. Sort d'autant plus choquant à nos yeux de «nouveaux observateurs"si l'on considère qu'à l'époque immédiatement précédente, la Grèce avait connu une période bien plus ouverte en ce qui concerne la"renommée"positive des femmes. Il suffit pour cela de nous reporter à l'âge d'or de la poésie lyrique où triomphe, parmi d'autres musiciennes ${ }^{19}$, Sappho de Lesbos, ce qui est la preuve évidente que, - et contrairement à ce qu'affirme le chœur de Médée quelques Grecques furent effectivement « dotées du chant inspiré de la lyre ».

\section{Une pédagogie chorale}

L'œuvre de Sappho est toujours d'actualité pour ce qui est de l'expression des émotions les plus intimes, et en particulier de la gamme subtile des sentiments qui se déploie entre la passion amoureuse et le dés-amour ${ }^{20}$. Si cet aspect des chants saphiques est remarquable au point de leur avoir conférél'immortalité, il ne devrait pas, cependant, occulter le fait que - c'est du moins ce que nous voudrions souligner - ces chants sont aussi le témoignage de la postérité d'une noble musicienne, reconnue officiellement, à un moment historique qui fut aussi bien bouillonnant du point de vue politique et guerrier qu'innovateur quant au développement des formes de pensée.

Nous nous reportons plus précisément à la deuxième moitié du VII siècle et aux premières décennies $d u \mathrm{VI}^{e}$ siècle av. J. C., c'est-à-dire à l'époque où l'on suppose que vécut Sappho, contemporaine des tyrans Myrsilos et Pittacos qu'elle eut comme ennemis en tant qu'aristocrate, et d'Alcée, le poète lyrique qui fut son allié et sonadmirateur. L'encyclopédie byzantine Souda ${ }^{21}$ et un papyrus ${ }^{22}$ où est conservée une partie de la biographie écrite au IV siècle par le péripatéticien Chamaléon nous apportent des informations très précises sur notre musicienne. 
13 Née dans l'île de Lesbos - on ne sait si c'est dans la ville d'Érésos ou dans celle de Mytilène - Sappho eut pour père Skamandrômnymos, ou bien Simon. Elle eut trois frères : Charaxos, qui s'en alla en Egypte « et pour une certaine Doricha dépensa la plus grande partie de sa fortune ", Eurygios et Larichos, le plus jeune et son préféré, dont la fonctiond'échanson au prytanée de Mytilène indique le rang aristocratique de la famille. Sappho épousa Kerkylas, " homme très riche », et en eut une fille qui porta le nom de sa mère, Kléis. Toujours d'après la tradition à laquelle nous faisons allusion, il faut ajouter à ces informations le niveau poétique exceptionnel atteint dans les neuf livres qu'elle écrivit, et l'invention du plectre - petit archetutilisé par les Grecs pour jouer des instruments à corde. Cette tradition fait aussi allusion aux aspects négatifs, tel le blâme pour ses inclinations sexuelles qui depuis portent son nom, son aspect physique plutôt ingrat, ou bien la passion destructrice qu'elle éprouva envers le jeune Phaon dont l'indifférence rendit sa lyre muette et provoqua sa disparition dans les eaux de la mer de l'île Leucade ${ }^{23}$.

14 Il est vrai qu'aujourd'hui, la plupart des renseignements auxquels nous venons de faire allusion sont contestés. Les informations sur la vie de Sappho paraissaient déjà à tel point contradictoires dans l'Antiquité qu'on est allé jusqu'à admettre l'existence de deux Sappho : l'une poétesse, l'autre courtisane ${ }^{24}$. Ainsi, dès l'origine, sa réputation va de pair avec la légende, comme si l'existence réelle d'une créatrice de sa catégorie fût difficile à admettre. C'est précisément à cet aspect singulier du personnage historique de Sappho que nous avons choisi de nous intéresser.

Étant donné l'époque et le contexte social où vécut Sappho, il est plus que probable qu'elle ait été mariée, mais ni son organisation quotidienne ni son économie domestique ne semblent avoir dépendude celui qui apparaît comme son époux, Kerkylas, peut-être parce qu'il mourut jeune ou peut-être parce qu'il était toujours en voyage comme Charaxos. En tous les cas, les fragments saphiques n'évoquent jamais le mari alors que les proches de la poétesse y trouvent une place significative: Sappho évoque sa chère fille, Kléis, qu'elle n'échangerait pas " contre toute la Lydie » ${ }^{25}$ et elle rapporte quelques conseils de sa mère :

...celle qui m'a mise au monde

...

$<$ me disait> (?) que dans sa jeunesse c'était un grand ornement si une jeune fille avait les cheveux enroulés dans un bandeau de pourpre...

...

de toutes les couleurs, venuede Sardes

Mais moi, pour toi, Kléis, je ne sais où aller prendre la mitre de toutes les couleurs... ${ }^{26}$.

16 Sappho prie aussi son frère Charaxos de retrouver la raison et de mettre un terme à sa vie capricieuse en quittant la dangereuse courtisane pour qui il perdit sa fortune :

ô Cypris, puisse-t-elle te trouver très amère Doricha, et ne plus pouvoir se vanter en disant que voilà la seconde fois qu'il s'en est allé vers un amour plein de désir nostalgique ${ }^{27}$.

La poétesse donne l'image d'une femme ${ }^{28}$ responsable de son entourage familial, ce qui nous éloigne de l'idée traditionnelle que nous avons de la femme grecque réduite au rôle de protégée sous tutelle au sein du foyer marital. Sappho fut une femme qui connut la réussite: les poteries de céramique portent souvent comme décor son élégante silhouette que l'on retrouve frappée sur les pièces de monnaie de Mitylène et d'Érèse ; plusieurs cités élevèrent des statues en son honneur. Savoir si cette renommée 
se traduisit en bénéfices économiques suffisants pour entretenir en permanence le train de vie somptueux de l'aristocratie à laquelle elle appartenait nous paraît difficile. Elle dut traverser, très probablement, des moments de largesse comme des moments de besoin au cours d'une vie marquée par le succès, mais aussi par l'exil dans la lointaine Sicile, où elle débarqua en aristocrate victime d'un régime tyrannique et dépourvue de tout. On remarquera toutefois que Sappho, pour qui le comble du bonheur est le mélange de richesse (ploûtos) et de vertu (arétas) ${ }^{29}$, se considéra particulièrement choyée par les divinités :

[Les Muses] qui m'ont faite honorée (timían)en me donnant leurs œuvres ${ }^{30}$..

18 Notre poétesse pourrait résumer en ces termes le fait d'avoir obtenu une reconnaissance socio-économique comparable à celle des anciens aèdes ${ }^{31}$; bien qu'ellemême situe une partie de son activité dans un cadre spatial bien spécifique de son sexe : « la demeure des servantes des Muses », dans laquelle le chant le plus opposé à la lyrique, le thrênos infernal, était interdit ${ }^{32}$. Nombreux sont les spécialistesqui ont cru reconnaître la fonction de cet espace et qui ont attribué à Sappho des professions diverses, en distinguant des domaines inséparables dans le monde grec. Ainsi la poétesse pouvait apparaître tantôt comme une éducatrice, tantôt comme lemembre distingué d'un groupe, ou cercle de femmes constituant l'équivalent d'une hétairie masculine, ou encore, comme la prêtresse d'un thiasos, d'une confrérie consacrée au culte d'Aphrodite ; des hypothèses traversées, dans l'ensemble, par ce qu'il est convenu d'appeler la «question saphique » (Sapphofrage), c'est-à-dire par un type d'analyse de l'érotique évoquée dans l'œuvre de Sappho, fondé sur l'opposition moderne entre homosexualité et hétérosexualité ${ }^{33}$.

19 Calamea apporté une précision fondamentale lorsqu'il a identifié le cercle des femmes réunies autour de Sappho comme un "chœur lyrique »: « ... plusieurs poétesses, spécialement en Grèce orientale, semblent avoir regroupé autour de leur personne un certain nombre de jeunes filles qui étaient à la fois leurs élèves et leurs compagnes ; sous leur direction, ces adolescentes se livraient, dans un cadre souvent cultuel, à une activité musicale qui confère à leur association une forme très proche, si ce n'est identique, de celle du chœur lyrique $»^{34}$. Très brièvement, nous pourrions définir cette institution du chœur lyrique comme un groupe de jeunes qui se consacrent à des représentations rituelles. Ces dernièress'appuient sur le chant et la danse et sont dirigées par un chorège auquel les jeunes du chœur sont unis par des liens d'ordre pédagogique et avec qui ils établissent des relations homo-érotiques ${ }^{35}$. En plus du rôle religieux que joue cette institution, l'étude consacrée par Calame aux fragments d'Alcman laisse voir une dimension politique importante dans la structure des chœurs lyriques de Sparte, formés par des adolescents représentatifs du corps dirigeant de la cité $^{36}$. La stricte dépendance des chœurs lyriques spartiates à l'égard du système politico-religieux officiel traduit, sans aucun doute, un caractère institutionnel que l'on peut détecter aussi dans le cercle saphique de Lesbos.

L'œuvre de Sappho fournit, en effet, des indices qui orientent nos recherches dans ce sens. En premier lieu, l'emploi de adikeîn, « commettre une injustice» et de philótes, " amitié », dans le contexte de l'Ode à Aphrodite composée par Sappho, révèle que la rupture des liens qui unissent l'un des membres du groupe aux autres est conçue non seulement comme une "infidélité » mais encore comme une véritable infraction juridique par rapport aux bases institutionnelles du groupe : «Trahir Sappho - affirme Calame - ce n'est pas seulement trahir un amour, c'est rompre des liens sanctionnés 
par un contrat ». En deuxième lieu, la dimension religieuse que revêt le chœur lyrique, apparemment comme lieu de communication entre la divinité et ses fidèles, est présente dans l'œuvre de Sappho à l'occasion d'invocations aux divinités. Enfin, la fonction pédagogique que le chef du chœur lyrique exerce - seul responsable de la préparation des jeunes qu'il dirige dans la représentation des compositions qu'il a crées - correspond parfaitement à la facette d'éducatrice de Sappho, essentielledans son personnage.

21 En définitive, si l'on retient l'interprétation de Calame, la figure de Sappho s'enrichit, puisque, d'une part, elle apparaît à la fois comme poétesse et chorège, et d'autre part, son œuvre s'inscrit dans l'espace des représentations publiques, au lieu d'être reléguée dans la célèbre "demeure des servantes des Muses" ${ }^{37}$. Ainsi interprétées, les principales hypothèses exprimées précédemment à propos de la fonction du cercle saphique cessent d'être contradictoires. De même, ce cercle, en tant qu'institution, se trouve totalement intégré dans l'espace public du système social qui l'encadre. Système certes fondé sur une certaine margination des femmes, mais à propos duquel l'œuvre de Sappho apporte inévitablement des informations, bien qu'une tradition bien attestée depuis l'antiquité, tend à ne reconnaître cette dimension informative que dans l'oeuvre poétique d'Alcée, contemporain et compatriote de Sappho.

\section{Le citoyen et la Muse}

Sappho et Alcée appartiennent à la même époque, même patrie et même classe sociale, allant même jusqu'à partager des orientations politiques identiques, à en juger par les noms qu'ils emploient pour désigner leurs ennemis communs et par la complicité respectueuse qu'Alcée utilise dans son œuvre lorsqu'il fait référence à Sappho. Quant à l'activité poétique proprement dite, tous deux s'y consacrent en partie lors de banquets - bien qu'il ne s'agisse pas des mêmes - et se rencontrent lors des concours des fêtes religieuses accueillies par les grands sanctuaires panhelleniques. Les plus célèbres à l'époque étaient certainement Olympie et Delphes en raisonde l'ampleur de leurs festivals. Mais les différentes cités de Lesbos possédaient aussi l'un de ces sanctuaires où, lors des concours, les sacrifices fastueux en l'honneur des dieux se mêlaient aux épreuves d'athlétisme et aux concours de poésie. Il s'agit d'un sanctuaire dédié à Héra, Zeus et Dionysos - la célèbre triade lesbienne - dont Alcée ${ }^{38}$ décrit l'extension et le caractère «commun" à tous les Lesbiens et dont Sappho narre les festivités dans la prière que nous citons ici

\footnotetext{
Dans mon sommeil naguère, tout près m'est apparu, ô souveraine Héra, ta forme gracieuse, telle que jadis l'aperçurent, appelée par leurs prières, les rois illustres, fils d'Atrée.

Car ayant achevé d'abord l'œuvre d'Arès, près du Scamandre aux flots rapides, en partant vers ces rivages-ci ils ne purent retourner chez eux

avant de t'avoir approchée de leurs prières toi-même et le grand Zeus et l'aimable fils de Thyona (Dionysos). Tels maintenant les citoyens t'offrent, suivant la tradition, de pures victimes, et les jeunes filles t'apportent un beau péplos, et avec elles les femmes, pressées nombreuses autour de ton autel ${ }^{39}$.
}

Les concours spectaculaires célébrés dans ce sanctuaire ont sans doute favorisé la renommée internationale des deux poètes de Lesbos. Or, le contraste entre l'univers présidé par Éros que présente Sappho dans son œuvre ${ }^{40}$ et les références à la guerre que l'on trouve constamment dans l'œuvre d'Alcée donne à penser que la vie de ces deux 
poètes ne se ressemblaient point. À partir de cette différence essentielle, Sappho est démise de son rôle detémoin de son temps :elle vivrait plongée dans une mer de sentimentalisme narcissique alors que l'on considère le soldat Alcée comme le chroniqueur de ce moment historique agité $e^{41}$.

La grande demeure resplendit d'airain. Arès a décoré toute la toiture de casques brillants, d'où pendent de blancs panaches en crinière de cheval, parure des têtes de guerriers. Suspendues tout autour, sur les chevilles qu'elles cachent, voici les brillantes cnémides, rempart contre le trait pénétrant; voici les corselets de lin neuf, et les creux boucliers empilés ; auprès d'eux, les glaives de fer de Chalcis, et les cottes, et les casaques de guerre : toutes choses qu'on ne peut oublier, dès que vient l'heure de la grande tâche ${ }^{42}$.

Habituellement on considère cette énumération littéraire d'armes comme la fidèle description d'un arsenal lesbien. Il est difficile, cependant, de ne pas voir la volonté d'héroïsation de ce poème, traversé par les idéaux du style épique dont la poésie d'Alcée comme celle de Sappho ${ }^{43}$ sont les héritiers directs. Or, avec la poétesse, nous nous trouvons exactement face à la situation inverse, ainsi que le démontrent les commentaires modernes à propos du poème qui reflète - en évoquant les fiançailles épiques d'Hector et Andromaque - le rite nuptial lesbien, et très précisément le moment où la future épouse est reçue chez le futur époux. Le poème, certainement un épithalame, s'achève ainsi dans la traduction de Pigeaud:

L'aulos au doux chant et la cithare se confondent, et le claquement des crotales, tandis que, de leur voix claire, les vierges chantent le chant sacré, et parvient jusqu'à l'éther l'écho divinement chanté ... et partout il y a, dans les rues, des cratères et des coupes...

Myrrhe et cannelle et encens se mêlent,

Et les femmes hululent, toutes celles qui sont âgées, et tous les hommes élèvent un cri de grâce, en invoquant Paon, l'Archer, qui tire au loin, à la belle lyre, et chantent un hymne en l'honneur d'Hector et d'Andromaque, semblable aux dieux ${ }^{44}$.

Reprenant Wilamowitz, Schadewaldt ${ }^{45}$ a nié que Sappho soit l'auteur de ce poème arguant qu'on n'y trouve pas "ce ton intime et personnel, féminin et sincère dans sa cordialité et son effusion » qui, d'après lui, la caractérise. À l'heure actuelle, aucun spécialiste ne discute le fait que Sappho ait été l'auteur de ce poème, mais on continue à dénier aux vers saphiques toute leur valeur de document reflétant un contexte social déterminé. Bowie ${ }^{46}$ propose un exemple à l'appui lorsqu'il affirme que dans le poème d'Hector et Andromaque « le mythe est raconté, non pas en tant qu'illustration, mais à cause du mythe lui-même ». Évidemment, les multiples objets qui font partie de la vie quotidienne des Lesbiennes nobles et qui sont rapportés dans les fragments saphiques, ne sont pas considérés, par nos érudits, aussi «réels» que les armes épiques qui fascinent Alcée. Et le milieu que Sappho décrit si attentivement ne serait que la recréation d'un monde "idéal » au sens d' "irréel », pur produit de son imagination. "L'amour est la seule réalité énergétique qui existe dans cette poésie sourde, démunie d'yeux et de nerfs, indifférente envers d'autres sujets ", affirme, par exemple, Lasso de la Vega ${ }^{47}$.

Il est certain que la tradition a conservé les textes amoureux de Sappho, sans doute, ceux que la poétesse avait préférés. Dans ce sens, il est juste de dire que la tradition a privilégié le penchant belliqueux d'Alcée laissant de côté sa dévotion à Éros, qu'il désigne comme «le plus terrible des dieux ${ }^{48}$ ». Ce sont les écrivains latins qui font état de cette tendance d'Alcée à « chanter l'amour des adolescents »"4. Ainsi Horace n'oublie pas les vers que Le citoyen de Lesbos a dédié au beau Lycus, mais c'est très clairement 
qu'il formule le partage des fonctions entre les deux poètes contemporains; partage sexué s'il en est, que la tradition a reproduite avec fidélité :

Combien j'ai été près de voir le royaume de la noire Proserpine [...] et Sappho se plaignant, sur ses cordes éoliennes, des jeunes femmes de son pays, et toi, chantant, avec des sons plus pleins sous ton plectre d'or, Alcée, les dures épreuves de la navigation, et celles de l'exil et celles de la guerre ${ }^{50}$.

Poursuivons. De la même façon que, dans l'œuvre d'Alcée, subsistent quelques fragments des passions amoureuses que le poète guerrier vécut et chanta, dans celle de Sappho, malgré la sélection drastique dont l'œuvre a souffert, on peut trouver quelques allusions au milieu socio-politique suffisamment explicites pour récuser la théorie qui veut que sa poésie évite tout sujet qui ne soit pas celui de l'amour et s'abstient particulièrement de toute référence politique. Ainsi, on ne s'attache jamais à évaluer l'écho historique de la célèbre plainte de Sappho qui ne parvient pas à obtenir un « diadème de Sardes » pour sa fille :

Mais moi, pour toi, Kléis, je ne sais où aller prendre la mitre de

toutes les couleurs ... mais pour le Mytilénien [Pittacos] ...

... avoir... des mitres ( ?) bariolées ...

... ces souvenirs de l'exil des fils de Cléanax ... la cité les conserve ;

ceux-ci terriblement se sont dispersés ...

Sappho dénonce ici clairement la récession économique dont souffrirent les nobles de Lesbos lorsque Pittacos - comme son contemporain Solon - limita, par exemple, les frais des funérailles aristocratiques ou interdit l'acquisition, habituelle parmi les nobles, de trousseaux lydiens ; évidente aussi la dénonciation de la situation politique qui conduisit beaucoup de Lesbiens à l'exil. Ce qui n'empêche pas que le fragment soit interprété de la façon suivante par Lesky : «la façon dont Sappho met en rapport les luttes des hommes avec un problème de parure alors à la mode pour les cheveux démontre délicieusement la relation exclusive et directe entre la poésie et la vie de cette femme $~^{51}$. De même, la plupart des commentateurs ne perçoivent qu'un reproche de femme abandonnée dans le fragment où la poétesse désapprouve la décision de la jeune fille qui s'éloigne de son groupe pour chercher l'amitié des femmes Penthilides, la famille danslaquelle le tyran Pittacos prit femme :

... il n'est pas permis, Mica, que tu ...

... mais moi je ne te laisserai pas faire

... même si tu as choisi l'amour d'une femme des Penthilides ${ }^{52}$.

29 Nous savons par Sappho elle-même qu'elle n'était pas la seule aristocrate à avoir un cercle de jeunes filles à Lesbos. Il arriva qu'une fois, la poétesse dut avoir amicalement recours à l'une de ces dames parce qu'elle avait besoin d'élargir son chœur de jeunes vierges à l'occasion d'une représentation dans une noce ${ }^{53}$. Mais dans ces cercles féminins, se retrouve l'inimitié existant entre les bandes de guerriers mentionnés plus haut, inimitié que la voix saphique exprime sous la forme de la malédiction allant même jusqu'à désavouer le talent poétique de ses rivales :

Morte tu seras gisante, sans que personne se souvienne de toi, <ni maintenant ni plus tard> non, personne pour l'avenir ; car tu n'as pas part aux roses de Piérie... ${ }^{54}$.

C'est avec une extrême dureté que le verbe saphique s'exprime contre une rivale considérée comme une piètre poétesse ou qu'il exprime le rejet absolu qu'une autre lui inspire : "Tout à fait alors rassasiés de Gorgô » ${ }^{55}$. De la même façon, Andromaque, la dirigeante d'un groupe féminin qui rivalise avec celui de Sappho, sera qualifiée par cette dernière de «rustre ou paysanne » (agroîotis), accusée peut-être même d'être 
plus sorcière que musicienne - si l'on prend en considération l'emploi du termethélgein, " enchanter, ensorceler ", sur lequel on aura à revenir -, insultes qui étaient loin d'être banales parmi ces chorèges archaïques, responsables autant de l'apparence et du charme de leurs jeunes filles que de leur adresse musicale :

Quelle paysanne a ensorcelé (thélgei) ton âme ..., enveloppée d'une robe de paysanne, qui ne sait pas tirer ses haillons sur ses chevilles ? ${ }^{56} .$.

31 Sappho critique ses rivales avec la même intransigeance qu'Alcée ses ennemis et, dans les deux cas, les insultes ne sont que le reflet de la tension sociale qui secoue l'île. Or, un auteur de la rigueur de Fränkel ${ }^{57}$, plongé dans la dimension esthétique de l'œuvre de Sappho, conclut de la façon suivante: "Dans la poésie de Sappho, le mouvement historique connaît un répit». Et c'est dans le même sens que Fernández Galianodéveloppe ses recherches sur la poétesse de Lesbos : «... pour Sappho, il n’y a pas de guerres, malgré le bruit des sabres qui parcourent l'Asie Mineure dans ces temps hasardeux; pas de révolutions [...], ni tâche professionnelle qui ne soit précisément rattachée à l'amour... On ne peut pas dire que Sappho écrive sur l'amour, qu'elle préfère l'amour, qu'elle se consacre à l'amour, non, elle est tout amour $\aleph^{58}$. Sappho s'est convertie en l'objet de son œuvre.

Mais nous serions injustes vis-à-vis de ces spécialistes modernes si nous ne signalions pas que la réduction de Sappho à l'univers délicat qu'elle-même recréa surgit déjà dans l'image laissée par son contemporain Alcée ${ }^{59}$ : "Oh divine (ágna)Sappho, ornée de violettes, au doux sourire...». Il ne fallut pas longtemps pour que la musicienne vénéréefût élevée - c'est-à-dire reléguée - à la catégorie symbolique de dixième Muse dans une épigramme attribuée à Platon ${ }^{60}$. Identifiée comme "Muse mortelle " à l'époque hellénistiquepar Antipatros de Sidon ${ }^{61}$, Sappho est même intégrée dans le cercle constitué par Aphrodite, Éros et Peitho, bien que cette reconnaissance soit une arme à double tranchant. Car,dans une autre épigramme ${ }^{62}$, le fait d'être reconnue comme "dixième dans la liste des charmantes Muses" l'exclut d'une liste de neuf génies du lyrisme archaïque qui commence à Pindare et s'arrête à Alcée.

Sans aucun doute, Boccace ${ }^{63}$ se fonde sur une tradition établie lorsqu'il fait de Sappho le pur symbole de la poésie. Tradition toujours alimentée de nos jours par des propositions forcées, telle celle de traduire le nom même de Sappho ${ }^{64}$ par « numinous ", la transformant ainsi pur esprit surhumain.En somme, le sort de la première poétesse d'Occident - celle qui fascina les Grecs avant même que, en plein triomphe de la démocratie athénienne, les femmes, non pas le féminin, disparaissent de la scène musicale -, c'est de survivre en tant que personnage historique à travers un processus évident de divinisation.

34 "La seule entre les femmes qui accompagnée de sa lyre rendit hommage à la beauté " ${ }^{65}$ se dés-humanise, donne son nom à une dixième Muse, devient un anonyme numen et partage aussi le milieu magique de l'ensorcellement ${ }^{66}$. Cette dernière caractéristique sera choisie par Plutarque lorsqu'il évoquera le chant saphique comme contre-exemple hédoniste de la parole sobre prononcée par la Pythie de Delphes :

Ne vois-tu pas quel charme possèdent les chants de Sappho, qui enchantent et séduisent (keloûnta kaì katathélgonta) ceux qui les écoutent?, tandis que la prophétesse apollinienne s'exprime « sans sourire, sans ornement, sans fard... » ${ }^{67}$

Or Sappho n'emploie le verbe thélgein que pour définir - comme nous venons de le préciser - le pouvoir « ensorcelant » qu'une autre poétesse, mauvaise à ses yeux, exerça sur l'une de ses élèves. Cependant, quelques siècles plus tard et sous la plume d'un 
prêtre d'Apollon,ce verbe désigne à lui seul la poésie saphique. Lorsque l'univers musical apollinien se dédouble entre la vérité prophétique et la mélodie enchanteresse, l'art de Sappho se situe dans le choix le plus inquiétant : il est ce piège inévitable que constitue pour l'ancien Grec l'association de la beauté et de la féminité à laquelle nous faisions allusion au début. En d'autres termes, les verbes ensorceler et séduire, thélgein et keleîn, identifient dangereusement notre Muse avec les Sirènes ${ }^{68}$, également connues comme les "enchanteresses ", (Keledónes), dont la "douce voix » ${ }^{69}$ constitue un référent irremplaçable dans le monde grec lorsqu'il s'agit d'évoquer le chant féminin. En effet, les termes choisis par Plutarque pour définir le grand pouvoir de séduction du chant de Sappho sont précisément ceux qui définissent le mieux le piège que représente l'« incanto» des sœurs infernales des Muses que sont les Sirènes ${ }^{70}$, "l'enchantement" exercé par ces contre-musiciennes jusqu'au jour où, incapables d'hypnotiser Ulysse, elles se précipitèrent dans la mer du haut d'un rocher. Á propos, ne raconte-t-on pas que Sappho fit de même dans l'île de Leucade, lorsque sa lyre se tut pour toujours à cause de la douleur de ne plus pouvoir séduire l'élu de son cœur. Et aussi la sage Sphix, Muse ailée de l'au-delà ${ }^{71}$, lorsque Edipe déchiffra son chant ravisseur...

$\mathrm{Au}$ terme de notre parcours, un groupe de voix féminines et ailées optant pour le suicide attire puissamment notre attention. Mais s'occuper d'elles serait s'occuper d'une autre musique : du chant sans lyre qui triompha dans le théâtre pendant le siècle d'or athénien, siècle inhospitalier s'il en fût pour de véritables créatrices comme Sappho.

\section{BIBLIOGRAPHIE}

BARKER Andrew, 2004, "Transforming the Nightingale: Aspects of Athenian Musical Discourse in the Late Fifth Century", in Penelope Murray et Peter Wilson (dir.), Music and the Muses. The Culture of Mousike in the Classical Athenian City, Oxford University Press, pp. 185-204.

BÉLIS Annie, 1999, Les musiciens dans l'Antiquité, Paris, Hachette.

BOARDMAN John, GRIFFIN Jasper et MURRAY Oswyn (dir.), 1988 (1986), The Oxford History of the Classical World, Madrid, Alianza Editorial.

BROWN Edwin L., 1991, "Sappho the numinous", ICS, XVI, 1-2, pp. 59-63.

CALAME Claude, 1977, Les choeurs de jeunes filles en Grèce archaïque, Roma, Edizioni

dell'Ateneo\&Bizzarri.

-, 1996, L'Éros dans la Grèce antique, Paris, Belin.

-, 2000, Le récit en Grèce ancienne, Paris, Belin.

DE MARTINO Francesco, 2006, Poetesse Greche, Bari, Levante-Editori.

DETIENNE Marcel, 1973 (1967), Maîtres de vérité, Paris, Maspéro.

FERNÁNDEZGALIANo Manuel, 1958, Safo, Madrid, Fundación Pastor de Estudios Clásicos. 
FRÄNKEL Hermann, 1993 (1962), Dichtung und Philosophie des frühen Griechentums, Madrid, Visor. GENTILI Bruno, 1996 (1984), Poesia e pubblico nella Grecia antica, Barcelona, Quaderns Crema. GREENE Ellen (dir.), 1996, Reading Sappho: Contemporary Approaches, Berkeley, University of Califonia Press.

IRIARTE Ana, Las redes del enigma. Madrid, Taurus, 1990.

-, 1993, "Le chant-miroir des Sirènes", VIII, 1-2, Métis, pp. 147-159.

-, 1997, Safo (siglos VII/VI a.C.), Madrid, Ediciones del Orto.

-, 2002, De Amazonas a ciudadanos, Madrid, Akal Ediciones.

LARDINOIS André, 2001, "Keening Sappho" in André Lardinois et Laura Mcclure (dir.), Making Silence Speak, Princeton and Oxford, Princeton University Press, pp. 75-92.

LASSO DE LA VEGA José, 1974, "La oda primera de Sappho", CFC, VI, pp. 9-93; VII, pp. 9-80.

LISSARRAGUE François, 1998, "Images du gynécée", in Paul Veyne, François Lissarrague et Françoise Frontisi-Ducroux, Les mystères du gynécée, Paris, Gallimard, pp. 155-198.

LORAUX Nicole, 1990, "Prólogo" à Ana Iriarte, Las redes del enigma, Madrid, Ediciones Taurus, pp. 9-15.

-, 1999, La voix endeuillée, Paris, Gallimard.

MANCINI Loredana, 2005, Il rovinoso incanto. Storie di Sirene antiche, Bologna, Il Mulino.

MARRY John D., 1979, "Sappho and the Heroic Ideal: érotos areté", Arethusa, 12.1, pp. 71-92.

MURRAY Penelope et WILSON Peter (dir.), 2004, Music and the Muses. The Culture of Mousike in the Classical Athenian City, Oxford University Press.

PAPADOPOULOU Zozie et PIRENNE-DELFORGE Vinciane, 2001, "Inventer et réinventer l'aulos : autour de la XIIe Pythique de Pindare", in Pierre Brulé et Christophe Vendries (dir.), Chanter les dieux. Musique et religion dans l'Antiquité grecque et romaine, Presses Universitaires de Rennes, pp. 37-57. PARADISO Annalisa, 1993, "Saffo, la poetessa", in Nicole Loraux (dir.), Grecia al femminile, RomaBari, Editore Laterza, pp. 39-72.

PARKER Holt, 1995, "Sappho's Public World", in Ellen Greene (dir.) Women Poets in Ancient Greece and Rome, University of Oklahoma Press, pp. 3-24.

PIGEAUD Jackie, 2004, Sappho. Poèmes, Paris, Payot\&Rivages.

REINACH Théodore, 1926, La musique grecque, Paris, Éditions d'aujourd'hui.

RISSMAN Leah, 1983, Love as War: Homeric Allusion in the Poetry of Sappho, Beiträge zur Klassischen Philologie, 157, Königstein.

RODRÍGUEZADRADOS Francisco, 1980, Lírica griega arcaica, Madrid, gredos.

sChADEWALDT Wolfgang, 1973 (1950), Sappho. Welt und Dichtung. Dasein in der Liebe, Buenos Aires, Eudeba.

schmitt PANTEl Pauline, 1997, La cité au banquet, Collection de l'École française de Rome, 157. SEGAL Charles, 1974, "Eros and Incantation: Sappho and Oral Poetry", Arethusa, 7.2, pp. 139-160. -, 1994, "La voce femminile e le sue contraddizioni : da Omero alla tragedia", Intersezioni, 14.1, pp. 71-92. 
SERGHIDOU Anastasia, 2001, "Athena Salpinx and the Ethics of Music", in Susan Deacy et Alexandra Villing (dir.), Athena in the Classical World, Leiden-Boston-Köln, Brill, pp. 57-74.

SVEMBRO Jesper, 1976, La parole et le marbre. Aux origines de la poétique grecque, Lund,

Studentlitteratur.

VÉRILHAC Anne-Marie et VIAL Claude, 1998, Le mariage grec du VIe siècle av. J.-C. à l'époque d'Auguste, Bulletin de correspondance hellénique, Supplément 32.

\section{NOTES}

1. Euripide, Médée 419-430. Traduction adaptée de celle de L. Méridier pour Les Belles Lettres, 1926.

2. Ainsi, les éditions commentées de Médée de L. Méridier 1926 : 138-139, G. Ammendola 1951 : 64,

E. Valgiglio $1972: 84$, A. Medina et J. A. López Férez $1977: 228$.

3. Homère, Odyssée XI, 436-438 et $456:$ « ... il est impossible de faire confiance aux femmes ».

4. Nous pensons, bien sûr, aux célèbres passages hésiodiques sur la création de Pandore de la Théogonie (561-612) et des Travaux (42-105).

5. Ainsi, M. A.Denys et L. Page 1938, Oxford : 104. Cf. A. Elliott $1969: 81$.

6. Valgiglio $1972: 85$.

7. Pour le débat sur la condition féminine sous le régime démocratique présenté par Euripide dans Médée : Iriarte 2002, 138-142.

8. Lois 654a.

9. À propos de la pratique musicale du peuple grec : Reinach $1926: 132-160$. Pour une perspective récente du phénomène culturel de la musique dans la vie grecque, avec une ample bibliographie : Murray et Wilson (dir.) 2004.

10. Reinach $1926: 134$.

11. Pour l'importance de la vocalité féminine dans la tradition littéraire grecque : Segal 1994.

12. Loraux 1999. Cf., à propos du caractère dissonant des voix attribuées par les tragiques aux personnages féminins, Loraux 1990 : 14-15.

13. Bélis $1999: 37-60$.

14. Voir néanmoins, le chapitre, riche en précisions, dédié à la polyvalence de l'aulós par Papadopoulou et Pirenne-Delforge 2001. Pour la flûte et le double rôle, politique et guerrier, d'Athéna, Serghidou 2001.

15. Lissarrague 1998 : 170-172. Cf. Barker 2004 : 197-198.

16. Pour l'opposition entre les deux instruments les plus populaires parmi les Grecs, voir la perspective classique de Reinach 1926:121: «À la lyre, instrument d'Apollon, organe de l'éthos, s'oppose l'aulos, plus spécialement approprié au culte dionysiaque, l'organe du pathos ». N. Loraux (1999 : 93-99) s'intéresse à cette opposition - voire incompatibilité - telle qu'elle est présentée dans la tragédie, le genre qui se pense comme une musique sans lyre.

17. Hormis la participation féminine aux chants et danses dans le contexte de quelques cérémonies comme le mariage: Vérilhac et Vial 1998: 320-325. Quant à la place, bien réglementée, des femmes des citoyens dans les festins civiques, voir Schmitt Pantel 1997 : 132-135.

18. Stehle 1997 : 117-118. Bélis 1999 : 52-53.

19. Pour une storia della letteratura greca al femminile actualisée avec lucidité, De Martino 2006.

20. «Elle a inventé le mal d'amour ", précise Pigeaud 2004 : 14. Sauf indication contraire, c'est l'édition que nous suivrons ici pour la version française des fragments saphiques.

21. s.v. «Sappho».

22. Oxyrhynchus 1800 . 
23. Pour un commentaire plus détaillé du débat sur la biographie et l'activité poétique de Sappho : Iriarte 1997.

24. Eliano, Varia historia, 12,19. La censure implicite dans ce dédoublement fut relevé dans Paradiso 1993.

25. Pigeaud 132 (= Voigt).

26. Pigeaud 98a (= Voigt). Nous reviendrons sur la suite de ce poème à forte résonance historique.

27. Pigeaud 5 (= Voigt).

28. La problématique de l'identité autorial de Sappho est abordée dans Calame 2000 : 30-31.

29. Pigeaud 148 (= Voigt).

30. Pigeaud 32 (= Voigt).

31. Gentili 1996 : 338-339. Pour la considération de ces Maitres de vérité: Detienne 1967. Cf., en ce qui concerne le rapport entre vérité et dépendance économique des poètes, Svembro $1976: 175$.

32. Pigeaud 150 (= Voigt). Pour le débat inspiré par ce fragment, Iriarte 1997 : 24-32.

33. Iriarte 1997 : 34-48.

34. Calame 1977 : I, 367-372. Thèse reprise dans Greene (dir.)1996.

35. À propos de l'homophilie dans la Grèce pré-classique, relevons le commentaire de Calame 1996 : 111 : «De même qu'il dessine l'espace réservé à la femme adulte entre oikos et lieux de culte, le processus d'éducation initiatique par l'amour situe l'homme entre salles de banquet et agora ".

36. Calame $1977: \mathrm{I}, 381-285$.

37. Cf. Stelhe (1997: 262-325) qui propose deux types d'audience pour Sappho: son cercle d'amies et les cérémonies religieuses.

38. Voigt 129.

39. Reinach 27 (Voigt 17).

40. Lardinois (2001: 75-92) souligne l'assujettissement de celle-ci aux «female speech genres » présents dans l'antiquité grecque dès l'œuvre homérique.

41. Iriarte 1997 : 59-69. Cf., dans la même perspective, Parker 2005.

42. Reinach, 137 (Voigt 140).

43. Marry 1979 : 271-292 ; Rissman 1983.

44. Pigeaud 44 (= Voigt).

45. Schadewaldt $1973: 30-31$.

46. Dans son chapitre sur la poésie lyrique Boardman, Griffin et Murray 1988 : 125-126.

47. 1974 : VII, 45-56.

48. Alcée, Voigt 327.

49. Cicéron, Tusc. IV, 33.

50. Horace, Odes, respectivement, livre I, XXXII et livre II, XIII, 21-28.

51. A. Lesky, Geschichte der griechischen literatur, Bern, 1963, s.v. «Sappho».

52. Pigeaud 71 (= Voigt).

53. C'est le sens accepté pour le 27 (Voigt). Ainsi Rodríguez Adrados 1980 :

«... pues vamos a una boda : bien..., así cuanto antes envíanos doncellas y que los dioses... ».

54. Pigeaud 55 (= Voigt).

55. Pigeaud 144 (= Voigt).

56. Pigeaud $57(=$ Voigt $)$.

57. Fränkel $1993: 181$ et 185.

58. Fernández Galiano 1958 : 8-9.

59. Voigt 384.

60. Anthologie Palatine IX, 506 ; Cf. IX, 66 et IX, 26..

61. Anthologie Palatine VII, 14.

62. Anthologie Palatine IX, 571. 
63. «Saphos » Buccolicum carmen.

64. C'est l'opinion retorse de Brown 1991.

65. Himérios, Discours 28, 2.

66. Segal $1974: 143$.

67. De Pythiae oraculis 397a. Cf. 763a-b, pour la création poétique de Sappho comme le produit de la possession apollinienne.

68. Homère Odyssée XII, 44. Katathélgo dit aussi le chant de la magicienne Circé : Odyssée X, 213.

69. Homère, Odyssée XII, 183, 187, 192, etc. Iriarte 1993.

70. À propos de cette parenté, Mancini 2005 : 52-75.

71. Iriarte 1990 : 131-144. 